\title{
Downregulation of Human Platelet Reactivity by Neutrophils Participation of Lipoxygenase Derivatives and Adhesive Proteins
}

\author{
J. Valles, ${ }^{\star *}$ M. T. Santos, ${ }^{\star *}$ A. J. Marcus, ${ }^{*}$ L. B. Safier, ${ }^{\star}$ M. J. Broekman, ${ }^{*}$ N. Islam, * H. L. Uliman, * and J. Aznar ${ }^{\star}$ \\ ${ }^{*}$ Divisions of Hematology/Oncology, Departments of Medicine and Pathology, Department of Veterans Affairs Medical Center, and \\ Cornell University Medical College, New York, New York 10010; and ${ }^{\ddagger}$ Research Center, University Hospital "La Fe," \\ Valencia 46009, Spain
}

\section{Abstract}

Unstimulated neutrophils inhibited activation and recruitment of thrombin- or collagen-stimulated platelets in an agonist-specific manner. This occurred under conditions of close physical cell-cell contact, although biochemical adhesion between the cells as mediated by $\mathbf{P}$-selectin was not required. Moreover, in the presence of monoclonal P-selectin antibodies that blocked biochemical platelet-neutrophil adhesion, thrombin-stimulated platelets were more efficiently downregulated by neutrophils. This suggested a prothrombotic role for $\mathbf{P}$-selectin under these circumstances. The neutrophil downregulatory effect on thrombin-stimulated platelets was amplified by lipoxygenase inhibition with 5,8,11,14-eicosatetraynoic acid. In contrast, the neutrophil inhibitory effect on platelets was markedly reduced by platelet-derived 12S-hydroxy-5,8-cis, 10-trans, 14-cis-eicosatetraenoic acid (12S-HETE), as well as by the platelet-neutrophil transcellular product, 12S,20-dihydroxy-5,8,10,14-eicosatetraenoic acid (12S,20-DiHETE), but not by another comparable metabolite, 5S,12S-dihydroxy-6-trans, 8-cis, 10-trans, 14-cis-eicosatetraenoic acid (5S,12S-DiHETE), or the neutrophil-derived hydroxy acid leukotriene $B_{4}$. The neutrophil downregulatory effect on thrombin-induced platelet reactivity was enhanced by aspirin treatment. This may represent a novel action of aspirin as an inhibitor of platelet function. These results provide in vitro biochemical and functional evidence for the thromboregulatory role of neutrophils and emphasize the multicellular aspect of hemostasis and thrombosis. (J. Clin. Invest. 1993. 92:1357-1365.) Key words: platelet activation and recruitment $\bullet$ serotonin release $\bullet$ thromboregulation $\bullet$ lipoxygenase metabolites $\cdot \mathbf{P}$-selectin

\section{Introduction}

Platelets and neutrophils are brought into proximity in a variety of circumstances during hemostasis, thrombosis, and the inflammatory response. Both cell types can modulate each others' reactivity by virtue of physical contact per se and biochemical interactions.

Parts of this study were presented at the annual meetings of the American Society for Clinical Investigation, Washington, DC, 2-5 May, 1990, and Seattle, WA, 3-6 May 1991, and were published in abstract form (1990, Clin. Res. 38:232a (Abstr.); and 1991, 39:254a (Abstr.).

Address correspondence to Dr. Aaron J. Marcus, Thrombosis Research Laboratory, Room 13028W, Department of Veterans Affairs Medical Center, 423 East 23rd Street, New York, NY 10010.

Received for publication 28 September 1992 and in revised form 6 April 1993.

J. Clin. Invest.

(C) The American Society for Clinical Investigation, Inc.

0021-9738/93/09/1357/09 \$2.00

Volume 92, September 1993, 1357-1365
Platelets or released products therefrom can modify the functional responsiveness of neutrophils, including such functions as chemotaxis, adhesion, aggregation, and release of neutrophil granule constituents (1). The complexities involved in studying effects of neutrophils on platelets are emphasized by recent contradictory reports in the literature. These range from neutrophil promotion of platelet reactivity (2-5) to neutrophil blockage of platelet responsiveness (6-12). The reasons for these differences are not completely understood, but must in part be attributable to methodology. This would include cell preparation, specific type and concentration of agonist, and use of either intact neutrophils or releasates derived therefrom (1).

We initiated a detailed study of platelet-neutrophil interactions as part of our interest in early cellular and biochemical events in thrombosis. The experimental system used allows for independent evaluation of platelet activation and recruitment as influenced by intact unstimulated neutrophils under conditions of close cell contact for a brief time interval. It was demonstrated that human neutrophils markedly decreased human platelet activation ( serotonin release) and recruitment (proaggregatory activity of cell-free releasates) induced by collagen or thrombin.

\section{Materials and Methods}

Blood collection, platelet processing, and labeling. $240 \mathrm{ml}$ of blood was collected by free flow in plastic tubing through a 15-gauge needle into $50-\mathrm{ml}$ polypropylene tubes (13-15). One tube contained $4.5 \mathrm{ml}$ of $3.8 \%$ sodium citrate in a total volume of $45 \mathrm{ml}$. Five others contained 6 $\mathrm{ml}$ of acid-citrate-dextrose $\left(\mathrm{ACD}^{1}(\mathrm{mM}): 38\right.$ citric acid, 75 sodium citrate, and 135 glucose) in a total volume of $46 \mathrm{ml}$. Blood was centrifuged at $200 \mathrm{~g}$ to obtain platelet-rich plasma (PRP). The PRP tube prepared with citrate (adjusted to a platelet count of $4-5 \times 10^{8} / \mathrm{ml}$ with platelet-poor plasma) was capped and maintained at $22^{\circ} \mathrm{C}$ under $5 \% \mathrm{CO}_{2}$-air, for use as assay system for platelet aggregation (13-15).

The PRP from the ACD-anticoagulated tubes was used to prepare $\left[{ }^{14} \mathrm{C}\right]$ serotonin (5-HT)-labeled washed platelets for use in the activation system $(13,14)$. Radiolabeled serotonin $\left(\left[{ }^{14} \mathrm{C}\right] 5-\mathrm{HT}\right)$ creatinine sulfate, $54 \mathrm{mCi} / \mathrm{mmol}$ (in $2 \%$ ethanol) was obtained from Amersham Corp. (Arlington Heights, IL). $0.4 \mathrm{nmol}$ was added per $\mathrm{ml} \mathrm{PRP}$ and the tube was mixed gently and left at room temperature for $60 \mathrm{~min}$ under $5 \% \mathrm{CO}_{2}$-air. The tube was inverted and placed under $\mathrm{CO}_{2}$-air once again during this interval. Uptake (average $96 \%$ ) was measured by counting $50 \mu \mathrm{l}$ of PRP for radioactivity compared with $50 \mu \mathrm{l}$ of plateletpoor plasma. Platelets were then washed as previously described (1315 ) and finally suspended in cold $0.9 \% \mathrm{NaCl}$ adjusted to $10 \% / \mathrm{ml}$. Imipramine ( $2.5 \mu \mathrm{M}$ final concentration) was added $1 \mathrm{~min}$ before the ago-

1. Abbreviations used in this paper: $\mathrm{ACD}, 38 \mathrm{mM}$ citric acid, $75 \mathrm{mM}$ sodium citrate, $135 \mathrm{mM}$ glucose; ASA, acetylsalicylic acid; DFP, diisopropyl fluorophosphate; 12S,20-DiHETE, 12S,20-dihydroxy-5,8, 10,14-eicosatetraenoic acid; 5S,12S-DiHETE, 5S,12S-dihydroxy-6trans,8-cis, 10-trans, 14-cis-eicosatetraenoic acid; EDRF/NO, endothelium-derived relaxing factor/nitric oxide; ETYA, 5,8,11,14-eicosatetraenoic acid; 12S-HETE, 12S-hydroxy-5,8-cis, 10-trans, 14-cis-eicosatetraenoic acid; 5-HT, serotonin, 5-hydroxytryptamine; LTB $_{4}$, leukotriene $\mathrm{B}_{\mathbf{4}}$. 
nist to prevent reuptake of released 5-HT. Activation was quantified as total released radioactivity by counting $50 \mu$ l cell-free releasate (see below) in $4 \mathrm{ml}$ Aquasol-2 (DuPont NEN Research Products, Boston, MA).

Preparation of neutrophil suspensions. The remaining blood sample in the ACD-anticoagulated tubes (after removal of PRP) was used for neutrophil preparation. This was accomplished by Dextran T500 sedimentation and subsequent centrifugation through Ficoll-Hypaque (d 1.077), as previously described (15). To ensure removal of hemoglobin after the erythrocyte lysis step, an additional wash of the neutrophils was carried out by resuspension in buffer and centrifugation. The surface of the pellet was then rinsed three times with saline. Neutrophils were finally resuspended in Hepes buffer (pH 7.45) (mM): 5 Hepes, $140 \mathrm{NaCl}, 5 \mathrm{KCl}, 1.29 \mathrm{CaCl}_{2}$, and $1.20 \mathrm{MgCl}_{2}$. Trypan blue exclusion by the neutrophils averaged $95 \%$. No platelets were seen on stained smears.

System for independent evaluation of platelet activation and recruitment. The two-stage in vitro system used (Fig. 1) has been previously described in studies with combined suspensions of platelets and erythrocytes $(13,14)$. In the present study, the phosphate-buffered saline was substituted with Hepes buffer (above) in the generating system (13). Platelets $\left(2 \times 10^{8} / \mathrm{ml}\right)$ or platelets plus neutrophils $\left(0.5-30 \times 10^{6} / \mathrm{ml}\right)$ in a total volume of $0.5 \mathrm{ml},\left(\mathrm{Ca}^{2+}\right.$ conc. $\left.1 \mathrm{mM}\right)$, were preincubated for $10 \mathrm{~min}$ at $37^{\circ} \mathrm{C}$, stimulated with collagen or thrombin, mixed by in verting three times, and centrifuged $(50 \mathrm{~s}, 13,000 \mathrm{~g})$. Within $1 \mathrm{~min}$ of stimulation, $50 \mu \mathrm{l}$ of the cell-free releasate obtained was scintillation counted to assess activation $\left(\left[{ }^{14} \mathrm{C}\right] 5-\mathrm{HT}\right.$ release $)$ or transferred $(50 \mu \mathrm{l})$ to the assay system (PRP) for assessment of proaggregatory activity (recruitment). The assay system consisted of $125 \mu \mathrm{l}$ of PRP containing 4-5 $\times 10^{8}$ platelets/ml, $100 \mu \mathrm{l}$ of Hepes buffer and calcium (final concentration $1 \mathrm{mM}$ ).

Control experiments demonstrated that supernatants of neutrophils alone, platelets alone, or platelet-neutrophil mixtures did not induce recruitment in the absence of platelet agonists. In addition, radioactivity measurements of supernates of ${ }^{14} \mathrm{C}[5-\mathrm{HT}]$-labeled platelets indicated that unstimulated neutrophils did not induce ${ }^{14} \mathrm{C}[5-\mathrm{HT}]$ release from platelets in the absence of a platelet agonist. Since collagen is a particulate agonist, it is eliminated from the supernatants to be tested, by the centrifugation procedure. When thrombin was used as agonist in the generating system (platelet activation), a fraction was

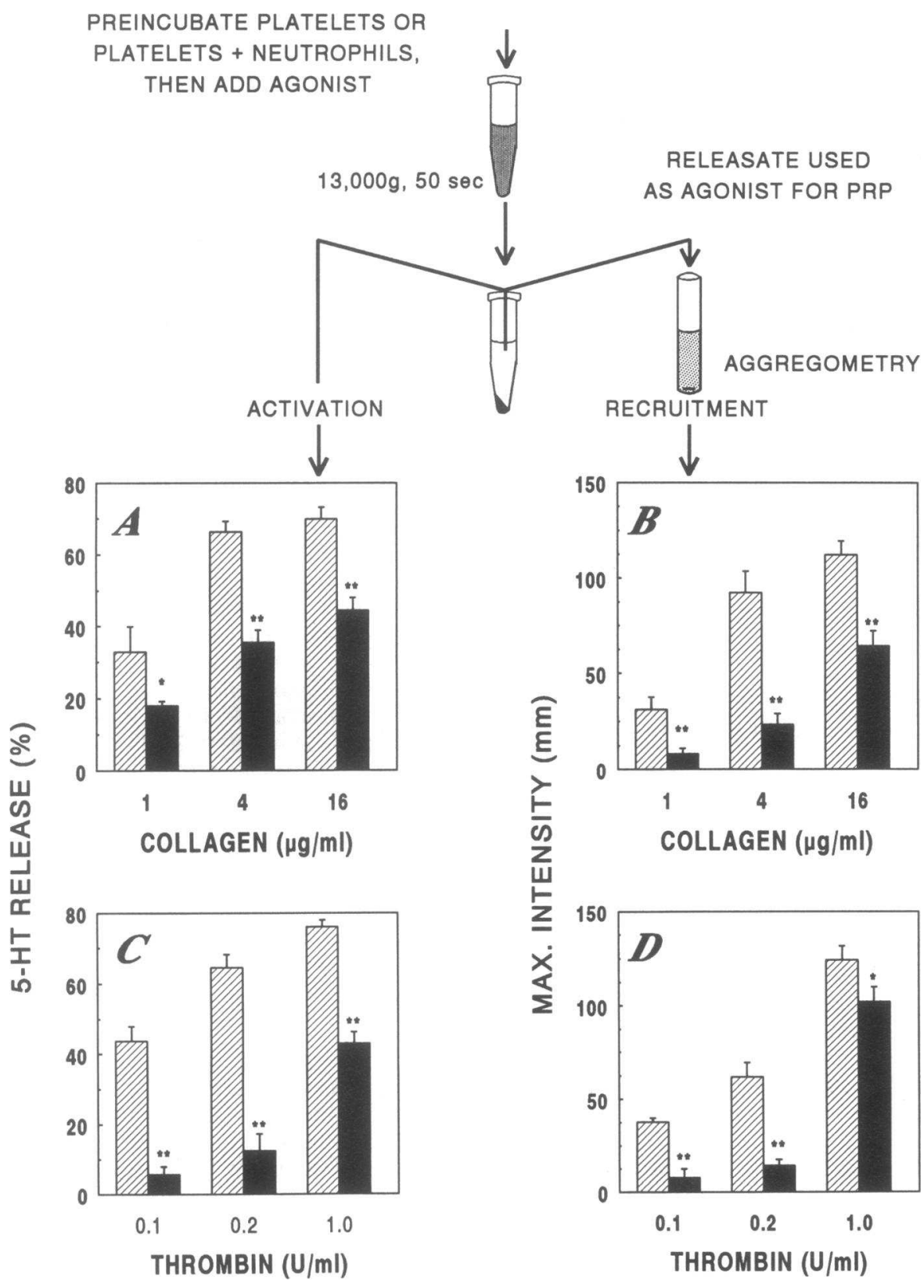

Figure 1. Effects of agonist concentration on inhibition of platelet activation by unstimulated neutrophils measured as percent serotonin (5-HT) release $(A$ and $C)$ and platelet recruitment $(B$ and $D)$. Platelets alone $\left(2 \times 10^{8} / \mathrm{ml}\right)(\square)$ or platelets plus neutrophils $\left(4 \times 10^{6} / \mathrm{ml}\right)(a)$ were stimulated with increasing doses of collagen or thrombin. Results are mean \pm SEM of paired samples $(n=6-9) ;{ }^{*} P<0.05,{ }^{* *} P$ $<0.005$. 
carried over to the PRP assay system (recruitment). At the highest concentration used $(1 \mathrm{U} / \mathrm{ml})$, a maximum of $0.18 \mathrm{U} / \mathrm{ml}$ could be theoretically transferred. This quantity of transferred thrombin never induced clotting in the PRP assay system within the time frame of the experiment (1-3 min). Neutrophils did not induce agonist degradation. This was demonstrated by an experiment in which thrombin was preincubated with or without neutrophils $\left(4 \times 10^{6} / \mathrm{ml}\right)$. An aliquot of the cell-free supernate demonstrated that the ability of thrombin to induce platelet 5-HT release was unimpaired.

Human thrombin (provided by Dr. John Fenton II) was used at 1 $\mathrm{U} / \mathrm{ml}$, and collagen (Hormon-Chemie, Munich, Germany) at $16 \mu \mathrm{g} /$ $\mathrm{ml}$ (unless otherwise specified for either agonist). Where used, aspirin (1 mM final concentration) or 5,8,11,14-eicosatetraynoic acid (ETYA) $(30 \mu \mathrm{M})$ were included during the 10 -min preincubation. For enzymatic removal of released ADP from the generating system, 2 $\mathrm{U} / \mathrm{ml}$ apyrase (grade I, Sigma Chemical Co., St. Louis, MO) was added during preincubation, 1 min before stimulation. To evaluate the effects of lipoxygenase metabolites, we added 12S-hydroxy-5,8-cis,10trans, 14-cis-eicosatetraenoic acid (12S-HETE), 5S, 12S-dihydroxy-6trans,8-cis, 10-trans, 14-cis-eicosatetraenoic acid (5S,12S-DiHETE) leukotriene $\mathrm{B}_{4}\left(\mathrm{LTB}_{4}\right)$ (Biomol Research Laboratories, Plymouth Meeting, PA), or 12S,20-dihydroxy-5,8,10,14-eicosatetraenoic acid (12S,20-DiHETE) (Cayman Chemical Co., Ann Arbor, MI) as sodium salts at $100 \mathrm{ng} / \mathrm{ml}$ during preincubation, $1 \mathrm{~min}$ before stimulation. Pharmacologic modulators of endothelium-derived relaxing factor/nitric oxide (EDRF/NO) used (16) were as follows: SOD (100 $\mathrm{U} / \mathrm{ml}), \mathrm{L}$-arginine $(10 \mathrm{mM})$, and hemoglobin $(20 \mu \mathrm{M})$ were all from Sigma. $N^{\mathrm{G}}$-Methyl-L-arginine $(500 \mu \mathrm{M})$ was from Calbiochem ( $\mathrm{La}$ Jolla, CA). $\mathrm{FeSO}_{4}(20 \mu \mathrm{M})$ was from Fisher (Pittsburgh, PA). All reagents were present during the preincubation period, except $N^{\mathrm{G}}$ methyl-L-arginine, which was previously incubated with the neutrophils for $60 \mathrm{~min}$. Hemoglobin was prepared by treatment with sodium dithionite ( 10 -fold excess), dialysis against water ( 3 changes in $2 \mathrm{~h}$ ), and storage in aliquots at $-70^{\circ} \mathrm{C}$. After thawing, the hemoglobin was oxygenated under a stream of oxygen and centrifuged at $13,000 \mathrm{~g}(2$ $\mathrm{min}$ ) to remove solids. Diisopropyl fluorophosphate (DFP) treatment of neutrophils was performed as previously described (15).

The murine monoclonal anti-P-selectin antibodies used were as follows: AC1.2 and GE12 (17) and G1, S-12, and W-40 (18-21). All antibodies were present during the $10-\mathrm{min}$ preincubation period at a concentration of $10-15 \mu \mathrm{g} / \mathrm{ml}$.

\section{Results}

Unstimulated neutrophils inhibit reactivity of platelets stimulated with collagen or thrombin

Effects of agonist concentration. Addition of collagen to a combined suspension of human platelets and neutrophils $(1 \mathrm{~min})$ resulted in a releasate (13) that demonstrated decreased platelet reactivity when compared with a releasate obtained from stimulated platelets alone (Fig. 1, $A$ and $B$ ). When the dose of collagen was increased from 1 to $16 \mu \mathrm{g} / \mathrm{ml}$, neutrophil inhibition of platelet 5-HT release (activation) relative to platelets alone varied from 46 to $36 \%$ (Fig. $1 A$ ). When the collagen-induced cell-free releasate was used as agonist to induce platelet recruitment in PRP (13), neutrophil-induced inhibition was pronounced (Fig. $1 B$ ).

Addition of thrombin to combined suspensions of platelets and neutrophils also resulted in a releasate with reduced 5-HT content, compared with releasates from platelets alone (Fig. 1 $C)$. An increase in dose from 0.1 to $1 \mathrm{U} / \mathrm{ml}$ thrombin decreased the percentage of neutrophil inhibition of 5-HT release from 87 to $43 \%$ (Fig. $1 C$ ). As with collagen, thrombin-induced platelet recruitment was lower in the presence of neutrophils (Fig. $1 D$ ). Inhibition of platelet reactivity by neutrophils demonstrated a steeper dose-response relationship for thrombin than collagen.

Effects of neutrophil number. Inhibition of platelet 5-HT release induced by collagen occurred at low concentrations of neutrophils $\left(0.5 \times 10^{6} / \mathrm{ml}\right)$ and plateaued in the physiological range $\left(4-8 \times 10^{6} / \mathrm{ml}\right)$ (Fig. $\left.2 A\right)$. The same phenomenon was observed when neutrophils inhibited platelet recruitment (Fig. $2 B)$. In addition to the washed system, neutrophils $\left(4 \times 10^{6} /\right.$ $\mathrm{ml})$ also inhibited platelet reactivity in platelet-rich plasma, stimulated by $16 \mu \mathrm{g} / \mathrm{ml}$ collagen $(41.1 \pm 0.9 \% 5-\mathrm{HT}$ release vs. $53.2 \pm 1.7 \%$ with PRP alone, mean \pm SEM, $P<0.01$ ). Platelet recruitment by cell-free supernates derived from PRP or PRP plus neutrophils also indicated neutrophil inhibition of platelet reactivity (PRP + neutrophils: $47.0 \pm 7.6 \mathrm{~mm}$; PRP alone:

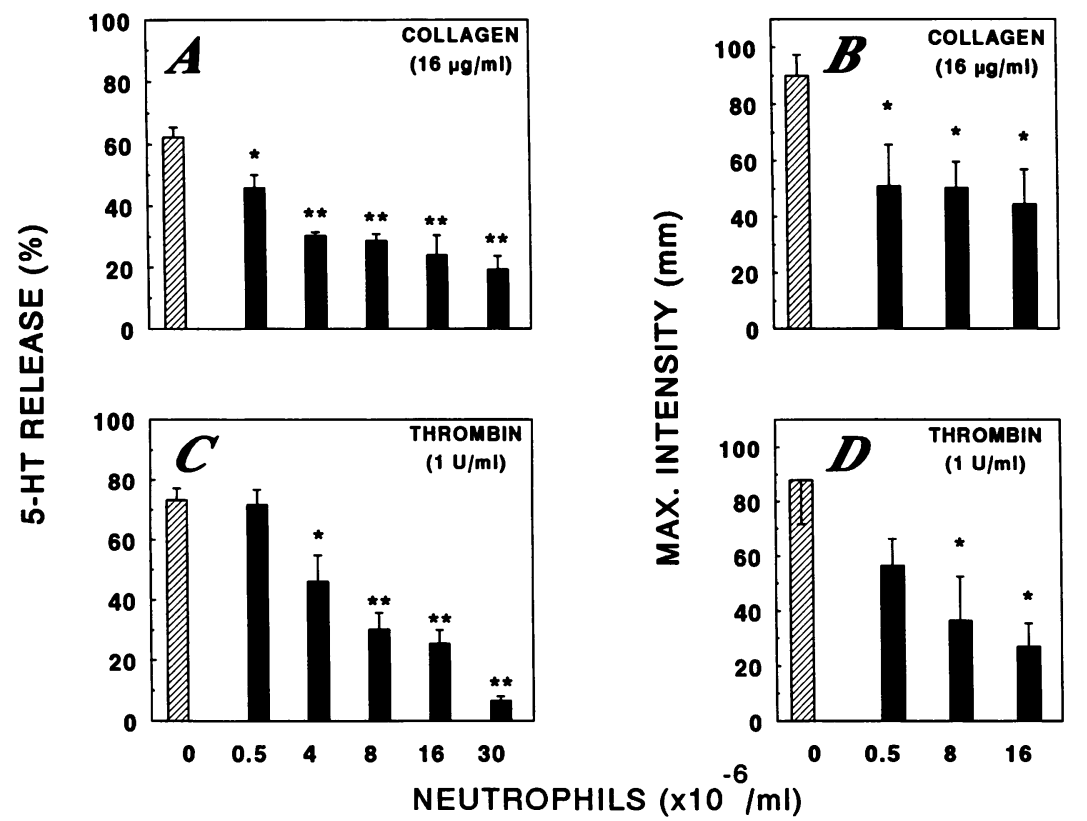

Figure 2. Effects of neutrophil number on inhibition of platelet activation measured as percent 5-HT release $(A$ and $C$ ) and platelet recruitment ( $B$ and $D$ ). Platelets $2 \times 10^{8} / \mathrm{ml}(\square)$. Results are mean \pm SEM of paired samples $(n=5) ;{ }^{*} P<0.025,{ }^{* *} P<0.001$. 


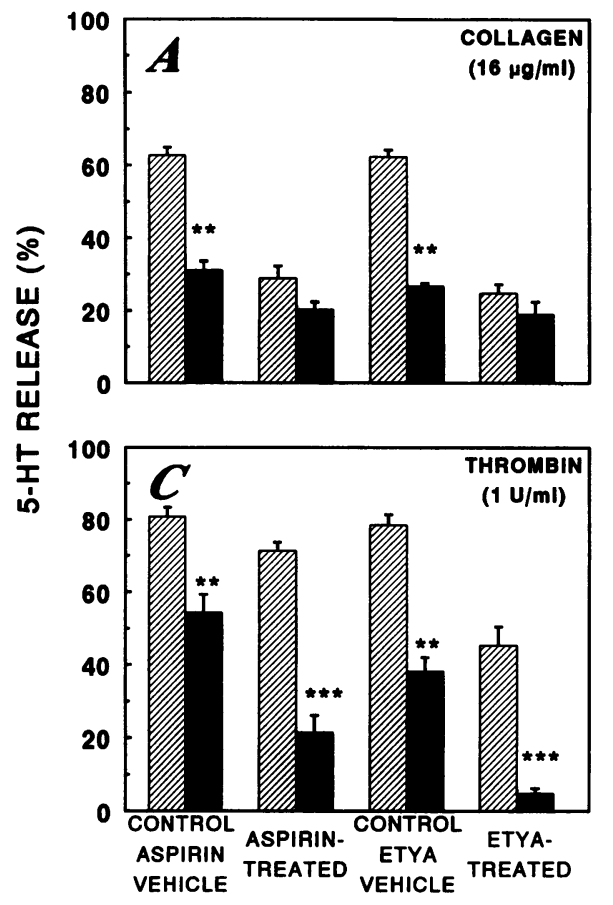

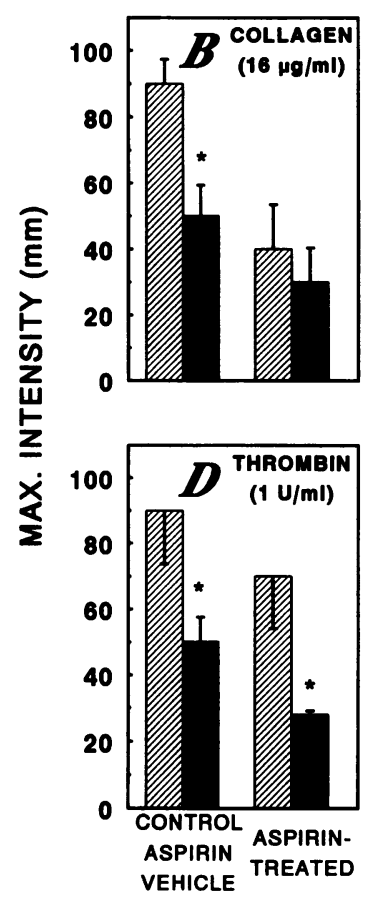

Figure 3. Effects of aspirin ( $1 \mathrm{mM})$ and ETYA ( 30 $\mu \mathrm{M}$, an inhibitor of both cyclooxygenase and lipoxygenase) on neutrophil inhibition of platelet activation (percent 5-HT release, $A$ and $C$ ) and platelet recruitment $(B$ and $D)$. Platelets alone $\left(2 \times 10^{8} / \mathrm{ml}\right)(\theta)$ or platelets plus neutrophils $\left(4 \times 10^{6} / \mathrm{ml}\right)(\square)$ were stimulated with collagen or thrombin. Aspirin- and ETYA-treated samples were compared with their respective vehicle controls. Results are mean \pm SEM of paired samples $(n=7-10) ;{ }^{*} P<0.01,{ }^{* *} P<0.005$, ${ }^{* * *} P<0.0001$
$74.5 \pm 3.4 \mathrm{~mm}, P<0.01)$. Similar results were obtained with 8 $\times 10^{6}$ and $16 \times 10^{6}$ neutrophils $/ \mathrm{ml}$.

In contrast to collagen, neutrophil-dependent inhibition of platelet activation with thrombin became significant at neutrophil concentrations in the physiological range. The inhibitory effect of neutrophils on thrombin-stimulated suspensions was highly dependent on neutrophil concentration (Fig. $2 C$ ). Neutrophil inhibition of platelet recruitment by thrombin-elicited cell-free releasates demonstrated a similar dependence on neutrophil number (Fig. $2 D$ ).

\section{Eicosanoid participation in inhibition of platelet activation by neutrophils}

Platelet eicosanoids such as $\mathrm{TXA}_{2}$ are rapidly released upon stimulation, and may have initiated the neutrophil inhibitory activity. This possibility was tested by pretreating platelets alone or platelet-neutrophil mixtures with aspirin during the preincubation period. With collagen, inhibition of platelet 5HT release by $4 \times 10^{6}$ neutrophils/ml was reduced by aspirin treatment (Fig. $3 A$ ). However, with higher numbers of neutrophils in the acetylsalicylic acid (ASA)-treated system, neutrophil inhibition of platelet activation induced by collagen was marked at $8 \times 10^{6}$ and $16 \times 10^{6}$ neutrophils $/ \mathrm{ml}$ (Table I).

In contrast to collagen, when the cells were stimulated with thrombin, neutrophil inhibition of platelet reactivity became far more effective after ASA-treatment (Fig. $3 C$ ). This inhibition was even more pronounced at higher neutrophil concentrations (Table I).

To extend the data obtained with the cyclooxygenase inhibitor ASA, we inhibited both cyclooxygenase and lipoxygenase with ETYA. In this instance as well, neutrophil inhibition of platelet 5-HT release (activation) was reduced with collagen as stimulus, but was potentiated with thrombin (Fig. 3). Results of experiments on recruitment in the setting of eicosanoid inhibition followed the same pattern as those described for activation (Fig. $3 B, D$ ).
Using $\left[{ }^{3} \mathrm{H}\right]$ arachidonate-labeled platelets in our activation-recruitment system (Fig. 1), we previously reported (13) that aspirin treatment of platelets alone resulted in a paradoxical reduction of $12 \mathrm{~S}-\mathrm{HETE}$ synthesis. This occurred during the short ( $<1 \mathrm{~min}$ ) time interval studied (13). The reduction in 12S-HETE was greater with thrombin $(81 \%)$ than collagen (42\%) (13). We interpret the data depicted in Fig. 3, $C$ and $D$, to indicate that a reduction in 12S-HETE formation is associated with an increase in the neutrophil inhibitory effect. This suggests that exogenous addition of lipoxygenase products could reduce neutrophil-dependent inhibition of platelet activation.

Effects of platelet and neutrophil lipoxygenase metabolites on neutrophil-dependent inhibition of platelet activation

Platelets alone and combined platelet-neutrophil suspensions were stimulated in the presence of exogenously added lipoxygenase metabolites. These included platelet-derived $12 \mathrm{~S}$ HETE, neutrophil-derived $\mathrm{LTB}_{4}, 12 \mathrm{~S}, 20$-DiHETE (formed by transcellular metabolism between stimulated platelets and un-

Table I. Platelet Serotonin Release Is Inhibited by Increasing Numbers of Unstimulated Neutrophils in an Aspirin-treated System

\begin{tabular}{ccc}
\hline $\begin{array}{c}\text { Neutrophils } \\
\left(10^{6} / \mathrm{ml}\right)\end{array}$ & Collagen $(16 \mu \mathrm{g} / \mathrm{ml})$ & Thrombin $(1 \mathrm{U} / \mathrm{ml})$ \\
\hline 0 & $33.92 \pm 2.47$ & $64.70 \pm 2.64$ \\
4 & $23.50 \pm 2.30$ & $35.23 \pm 5.10^{\ddagger}$ \\
8 & $16.55 \pm 8.80^{*}$ & $15.00 \pm 7.50^{\ddagger}$ \\
16 & $11.36 \pm 8.80^{*}$ & $3.82 \pm 1.20^{\ddagger}$ \\
\hline
\end{tabular}

Serotonin release $(\%)$ is expressed as mean \pm SEM, obtained from paired samples $(n=7)$. For the comparison platelets alone $\left(2 \times 10^{8} /\right.$ ml) vs. platelets + neutrophils: ${ }^{*} P<0.005$ and ${ }^{\ddagger} P<0.001$. 
stimulated neutrophils), and 5S,12S-DiHETE (formed by transcellular metabolism between stimulated platelets and stimulated neutrophils) $(22,23)$.

With collagen as agonist, none of the lipoxygenase metabolites modified platelet activation (5-HT release) in the plateletneutrophil system (Table II). In sharp contrast, with thrombin, the 12-lipoxygenase products 12S-HETE and 12S,20-DiHETE, but not neutrophil 5-lipoxygenase metabolites, reduced the neutrophil inhibitory effect (Table II). These results support the concept that 12-lipoxygenase products reduce inhibition of thrombin-activated platelets by neutrophils.

\section{Effects of enzymatic removal of released platelet ADP}

We examined possible participation of neutrophil ecto-ADPase(s) activity $(24,25)$ in neutrophil inhibition of platelet reactivity. Platelets alone or platelet-neutrophil suspensions were stimulated in the presence or absence of the ADPase, apyrase $(2 \mathrm{U} / \mathrm{ml})$. With platelets alone, apyrase reduced 5-HT release in collagen-, but not in thrombin-stimulated samples (Fig. 4). This indicated that under these conditions, removal of released platelet ADP reduced collagen-induced 5-HT release (Fig. 4, bar $a$ vs. $c$ ), but did not significantly inhibit thrombininduced 5-HT release (Fig. 4, bar $a^{\prime}$ vs. $c^{\prime}$ ). Therefore, with thrombin, the inhibitory effect of neutrophils did not result from neutrophil ecto-ADPase. This is shown in Fig. 4 where with thrombin, neutrophils eliminated a total of $41 \% 5-\mathrm{HT}$ release ( $\operatorname{bar} a^{\prime}$ minus bar $b^{\prime}$ ), which was paralleled by a decrease of $42 \% 5$-HT release due to non-ADP-dependent mechanisms ( $\operatorname{bar} c^{\prime}$ minus bar $d^{\prime}$ ).

However, similar calculations with collagen as agonist, assuming that ADP-dependent and non-ADP-dependent 5-HT release are additive, indicate a possible contribution of neutrophil ADPase. Here, the neutrophil inhibitory effect results in a loss of 34\% 5-HT release (bar $a$ minus bar $b$ ), whereas nonADP-dependent mechanisms cause a decrease of only $24 \%$ 5-HT release (bar $c$ minus bar $d$ ). The difference, a loss of $10 \%$ 5-HT release, is likely due to removal of released platelet ADP by neutrophil ecto-ADPase. The fraction of the total inhibitory effect potentially related to neutrophil ecto-ADPase (10/34 or $29 \%$ ) is therefore significant in the case of collagen, although not the major cause of neutrophil-dependent inhibition of platelet reactivity in this experimental setting.

Table II. Effects of Lipoxygenase Metabolites on Inhibition of Platelet Serotonin Release by Unstimulated Neutrophils

\begin{tabular}{lcc}
\hline & Collagen $(16 \mu \mathrm{g} / \mathrm{ml})$ & Thrombin $(1 \mathrm{U} / \mathrm{ml})$ \\
\hline Control & 46.8 & 30.8 \\
12S-HETE & 44.3 & $18.2^{*}$ \\
12S,20-DiHETE & 42.7 & $11.4^{*}$ \\
5S,12S-DiHETE & 52.1 & 32.9 \\
LTB $_{4}$ & 54.5 & 32.8 \\
\hline
\end{tabular}

Lipoxygenase metabolites were added at $100 \mathrm{ng} / \mathrm{ml}$. Data were obtained from paired samples $(n=4)$ of platelets alone $\left(2 \times 10^{8} / \mathrm{ml}\right)$ and platelets plus neutrophils $\left(4 \times 10^{6} / \mathrm{ml}\right)$ and are expressed as a percentage: $100 \times\left[1-\left(5-\mathrm{HT}_{\text {platelets +neutrophils }}\right) /\left(5-\mathrm{HT}_{\text {platelets alone }}\right)\right]$. The inhibition of platelet serotonin release by neutrophils was significantly reduced by $12 \mathrm{~S}$-HETE and $12 \mathrm{~S}, 20$-DiHETE, ${ }^{*} P<0.01$, in the case of thrombin-stimulated samples.

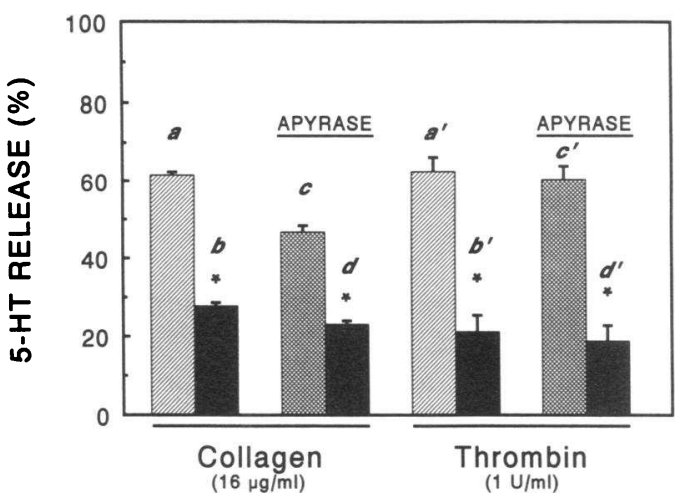

Figure 4. Effects of enzymatic removal of released platelet ADP on neutrophil inhibition of platelet activation (5-HT release). Platelets alone $\left(2 \times 10^{8} / \mathrm{ml}, a, c, a^{\prime}\right.$, and $\left.c^{\prime}\right)$ or platelets plus neutrophils (4 $\times 10^{6} / \mathrm{ml}, b, d, b^{\prime}$, and $\left.d^{\prime}\right)$ were stimulated with collagen or thrombin in the absence $\left(a, b, a^{\prime}\right.$, and $\left.b^{\prime}\right)$ or in the presence $\left(c, d, c^{\prime}\right.$, and $\left.d^{\prime}\right)$ of the ADPase apyrase $(2 \mathrm{U} / \mathrm{ml})$. Results are mean \pm SEM of paired samples $(n=4) ;{ }^{*} P<0.001$ for the comparisons $a$ vs. $b, c$ vs. $d, a^{\prime}$ vs. $b^{\prime}, c^{\prime}$ vs. $d^{\prime} ; P<0.001$ for $a$ vs. $c ; P<0.01$ for $b$ vs. $d ; a^{\prime}$ vs. $c^{\prime}$ and $b^{\prime}$ vs. $d^{\prime}$ not significant.

\section{Neutrophil inhibition of platelet reactivity is independent of} $E D R F / N O$ synthesis or serine protease activity

Neutrophils have been reported to produce EDRF/NO (2628 ), which can inhibit platelet responsiveness (9-12). We therefore evaluated effects of compounds known to modulate NO formation and/or activity (16). Platelets or platelet-neutrophil suspensions, either ASA treated or untreated, were stimulated with collagen $(16 \mu \mathrm{g} / \mathrm{ml})$ or thrombin $(1.0 \mathrm{U} / \mathrm{ml})$. This was done in the presence or absence of SOD, L-arginine, hemoglobin, $\mathrm{FeSO}_{4}, N^{\mathrm{G}}$-methyl-L-arginine, and L-arginine plus $N^{\mathrm{G}}$ methyl-L-arginine (data not shown). These pharmacologic modulators of EDRF/NO caused no variations in 5-HT release and therefore no changes in the neutrophil inhibitory effect on platelet activation under these conditions. Thus, in our activation-recruitment system, NO does not account for the neutrophil inhibitory effect on platelet reactivity.

Activated neutrophils release serine protease(s) that can modify platelet function. Although we were working with unstimulated PMN, we treated neutrophils with the cell-penetrating serine protease inhibitor, DFP. DFP-treated and -untreated neutrophils in combined suspension with platelets in the activation system were compared. Neutrophil inhibition of platelet activation was unaffected by DFP treatment (data not shown). Therefore, with unstimulated neutrophils, the inhibition of platelet activation was not mediated by serine proteases.

\section{P-selectin-dependent platelet-neutrophil adhesion limits neutrophil inhibition of platelet reactivity}

P-selectin [platelet activation-dependent granule-external membrane protein (29) (PADGEM), a granule membrane protein of $M_{r} 140,000$ (30) (GMP-140), CD 62 ] belongs to the selectin family of adhesive proteins (29-31). P-selectin mediates adhesion of activated platelets to neutrophils and monocytes $(17,20,21,32-34)$ and subsets of lymphocytes (35). We used specific murine monoclonal anti-P-selectin antibodies to determine whether a P-selectin-mediated platelet-neutrophil adhesion step was required for neutrophil inhibition of platelet 
reactivity. The antibodies GE12 (17) and G1 $(20,21)$ inhibit P-selectin-mediated neutrophil adhesion (30). Control antiP-selectin antibodies AC1.2 (17), S12 (20,21), and W40 (18) do not inhibit platelet-neutrophil adhesion.

Antibodies GE12 or G1 had no effect on the activation of platelets alone (Figs. 5 and 6). However, in the presence of these antibodies (which inhibit platelet-neutrophil adhesion), the neutrophil inhibitory effect on platelet activation was actually enhanced. This enhancement was more pronounced in thrombin- than in collagen-stimulated cell mixtures. With collagen as agonist, neutrophil-dependent platelet inhibition increased from 61 to $68 \%$. The percentage inhibition was calculated as 5-HT released by: $100 \times[$ (platelets alone - platelets plus neutrophils)/platelets alone]. Similarly in the presence of G1, platelet inhibition by neutrophils increased from 40 to $56 \%$. With thrombin, this trend was even more obvious. Neutrophil-dependent platelet inhibition rose from 67 to $90 \%$ upon addition of GE12, and from 23 to $52 \%$ with G1 (Figs. 5 and 6 ). The enhancing effect of these antibodies is even more apparent if 5-HT release of platelet-neutrophil mixtures in the absence and presence of adhesion-blocking antibodies is compared. If 5-HT release in the absence of these antibodies is considered to be $100 \%$, then with collagen as agonist, inhibition increased over controls by 22 and $24 \%$ with GE12 and G1, respectively.

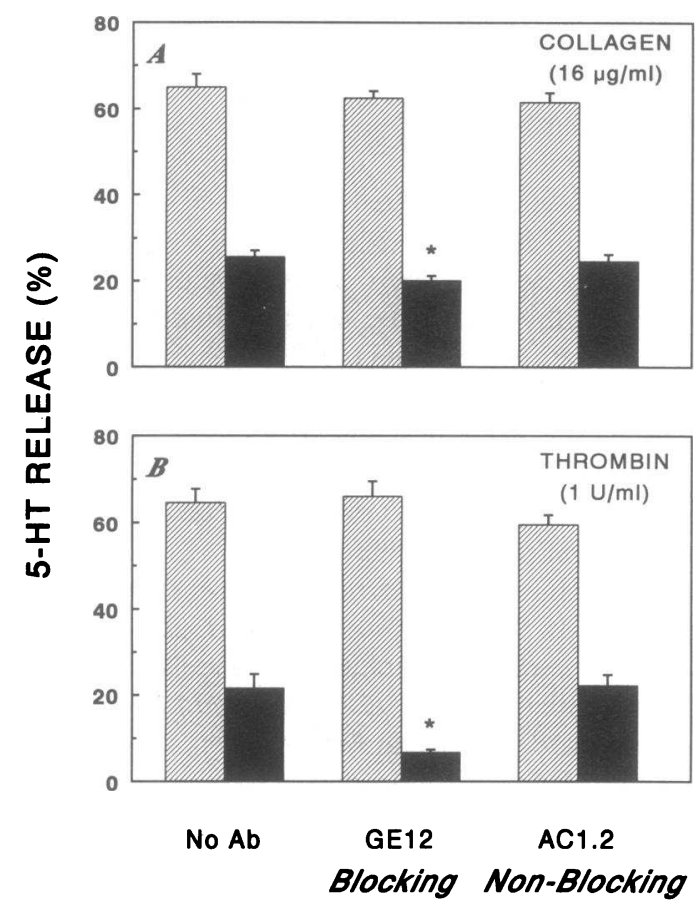

Figure 5. A specific monoclonal antibody against P-selectin, which inhibits platelet-neutrophil adhesion (GE12), enhances neutrophil inhibition of platelet activation (5-HT release), whereas a different specific monoclonal antibody against P-selectin, which does not inhibit platelet-neutrophil adhesion (AC1.2), was without effect. Platelets alone $\left(2 \times 10^{8} / \mathrm{ml}\right)(\square)$ or platelets plus neutrophils ( 8 $\left.\times 10^{6} / \mathrm{ml}\right)(\square)$ were stimulated with collagen or thrombin. Results are mean \pm SEM of paired samples $(n=8) ;{ }^{*} P<0.001$ for both the comparison of platelets plus neutrophils between GE12 and control (no antibody), as well as between antibody GE12 and antibody AC1.2. In all cases $P<0.0001$ for the difference between 5-HT release from platelets alone and from platelets plus neutrophils.

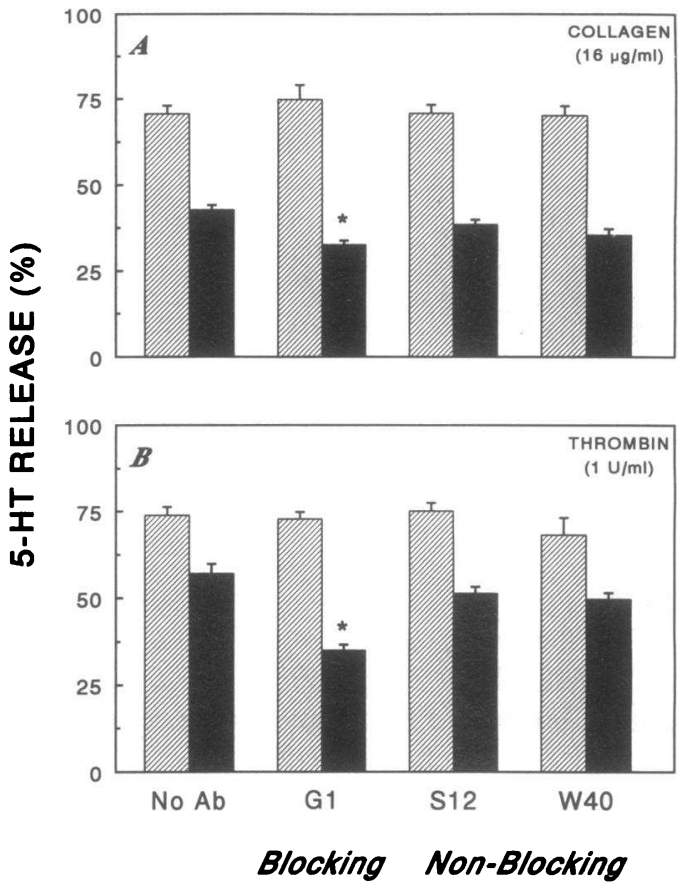

Figure 6. Results comparable to those shown in Fig. 5 were obtained with another group of antibodies against P-selectin. Antibody G1, which inhibits adhesion between activated platelets and unstimulated neutrophils, enhances neutrophil inhibition of platelet activation (5HT release). Antibodies S12 and W40, which do not inhibit platelet-neutrophil adhesion had no effect on neutrophil inhibition of platelet activation. Experimental conditions and $P$ values are the same as for Fig. 5.

With thrombin the inhibition increased 69 and 39\%, respectively. In contrast, the monoclonal anti-P-selectin antibodies $\mathrm{AC1.2,} \mathrm{S12,} \mathrm{or} \mathrm{W40} \mathrm{(which} \mathrm{do} \mathrm{not} \mathrm{affect} \mathrm{platelet-neutrophil}$ adhesion ) essentially had no influence on neutrophil inhibition of platelet activation (Figs. 5 and 6). These data indicated that a platelet-neutrophil adhesion step was not a prerequisite for the neutrophil to block platelet reactivity. In fact inhibition was enhanced in the presence of antibodies that blocked adhesion.

\section{Discussion}

Unstimulated human neutrophils strongly downregulated human platelet responsiveness during the first minute of stimulation by platelet agonists. This was demonstrated in an in vitro system (Fig. 1), which independently evaluates platelet activation and recruitment. This method may serve as an in vitro model for early hemostatic and thrombotic events (13). After the agonist is added, platelets and neutrophils are brought into close contact, and then the cells are removed. The supernatant fluid containing the products released from the cells is then used as a probe for platelet recruitment and tested biochemically for platelet activation. Thus, our system evaluates very early events, within 1 min of stimulation.

Since platelet agonists are more likely to be available during the early phase of thrombus formation, we chose collagen and thrombin for these studies. Choice of agonist for the stimula- 
tion of the cell mixture is a major determinant of the end result. In agreement with our data, addition of a platelet agonist to platelet-neutrophil mixtures resulted in neutrophil inhibition of platelet reactivity (8-10). However, with some neutrophil agonists, platelet reactivity in combined suspensions was promoted $(4,5)$.

The experimental results presented herein indicate in addition that neutrophil inhibition of platelet reactivity induced by collagen or thrombin was stimulus specific. At similar levels of activation of platelets alone, neutrophil inhibition of 5-HT release was greater in samples stimulated with thrombin than with collagen (Fig. $1, A$ and $C$ ). Furthermore, at low neutrophil concentrations, inhibition was more efficient with collagen than with thrombin (Fig. 2). Differences between these platelet agonists were also observed in eicosanoid regulation of platelet-neutrophil interactions. At the same neutrophil number, aspirin enhanced the neutrophil effect with thrombin but reduced it with collagen (Fig. 3 ). In addition, 12S-HETE had a regulatory role in thrombin- but not in collagen-stimulated samples (Table II). Last, P-selectin antibodies, which block adhesion, had much more pronounced effects with thrombin as platelet agonist, than with collagen (Figs. 5 and 6).

Neutrophil inhibition of platelet reactivity as observed in our in vitro system could have implications for comprehension of early events in hemostasis and thrombosis. The data suggest that neutrophils have the capacity to modulate initiation and propagation of a developing platelet thrombus.

As mentioned above, in the setting of aspirin treatment, the neutrophil inhibitory effect was reduced with collagen at physiological neutrophil concentrations $\left(4 \times 10^{6} / \mathrm{ml}\right.$, Fig. $\left.3 A\right)$. This effect was reversed at higher neutrophil concentrations (Table I). This suggested the presence of two mechanisms of neutrophil control: aspirin-sensitive and aspirin-insensitive. The aspirin-sensitive mechanism seems more efficient since lower quantities of neutrophils were required.

Treatment of combined platelet-neutrophil suspensions containing $4 \times 10^{6}$ neutrophils/ml with ETYA (inhibiting both cyclooxygenase and lipoxygenase) in samples stimulated with collagen yielded data similar to that obtained after aspirin treatment (Fig. $3 \mathrm{~A}$ ). This indicated that lipoxygenase metabolites were not involved in neutrophil inhibition of platelet reactivity in collagen-stimulated cells. The concept was verified by the inability of exogenously provided lipoxygenase products to alter neutrophil inhibition of platelet activation (Table II).

In contrast to the above results with collagen, neutrophil inhibitory effects on platelet reactivity were enhanced after thrombin stimulation of the aspirin-treated system (Fig. $3 \mathrm{~B}$ ). At the 1 min time point in our activation system (Fig. 1), aspirin treatment led to reduced 12S-HETE production (13). The dual cyclooxygenase and lipoxygenase inhibitor, ETYA further depleted the quantity of 12S-HETE formed. In this instance, neutrophil inhibitory activity of platelet activation with thrombin was even further enhanced (Fig. $3 \mathrm{~B}$ ). It can therefore be inferred that 12-lipoxygenase metabolites reduce thrombin-induced neutrophil inhibitory activity. This, in fact, did occur when 12-lipoxygenase metabolites were exogenously added to the thrombin-stimulated system (Table II).

Our data with thrombin provide yet another example of participation of platelet lipoxygenase metabolites in interactions involving other cell types, i.e., neutrophils (23). Downregulation of the neutrophil inhibitory effect was not abrogated by transcellular metabolism of platelet $12 \mathrm{~S}-\mathrm{HETE}$ to $12 \mathrm{~S}, 20-\mathrm{Di}-$ HETE via $\omega$-hydroxylation by unstimulated neutrophils (22). In contrast, this property of $12 \mathrm{~S}-\mathrm{HETE}$ was no longer demonstrable with 5S,12S-DiHETE generated from platelet 12SHETE via 5-lipoxygenation by stimulated neutrophils (23) (Table II).

Since they reduce neutrophil inhibition of platelet reactivity, 12-lipoxygenase products are "prothrombotic" in thrombin-stimulated combined cell suspensions. The early ASA-induced reduction in 12-HETE formation can therefore be regarded as a beneficial effect of this compound, occurring in addition to cyclooxygenase inhibition. Furthermore, the enhancement of neutrophil inhibition in ASA-treated samples stimulated with thrombin may also be a part of the beneficial therapeutic effect of aspirin when platelet activation by thrombin occurs in vivo (Fig. $3 \mathrm{~B}$ ).

Several additional mechanisms could have been responsible, at least in part, for neutrophil inhibition of platelet reactivity as demonstrated in our system. These included neutrophil ADPase(s), serine proteases, and EDRF/NO formation. The results obtained rule out participation of neutrophil EDRF/ $\mathrm{NO}$ and serine proteases since no variations in 5-HT release were found in the presence or absence of pharmacologic modulators of EDRF activity or after treatment with the cell-penetrating protease inhibitor DFP. Neutrophil ADPase activity was a factor only in samples stimulated with collagen, but not when thrombin was used as agonist. This may reflect the observations that stimulation of platelets by high doses of thrombin does not depend on platelet-released ADP while collagen stimulation of platelets does (36).

The fact that most of the above mechanisms were not involved in our results is probably a reflection of the following: $(a)$ we studied unstimulated neutrophils; $(b)$ the platelets were highly stimulated with specific agonists; $(c)$ maximal cell contact was facilitated; and $(d)$ duration of platelet-neutrophil contact was brief in order to simulate early in vivo thrombotic events.

Upon cell activation, the adhesive protein P-selectin is expressed on the platelet or endothelial cell surface $(17,30,31)$, where it mediates adhesion of neutrophils and monocytes. It has been suggested that P-selectin may be responsible for initial adhesion of neutrophils at sites of vascular injury, leading to leukocyte rolling $(20,37,38)$. In vivo, P-selectin may play a central role in leukocyte accumulation within thrombi (39).

It was important to discern whether direct platelet-neutrophil adhesion was necessary for the inhibitory effect of neutrophils on platelets to occur. This was examined with specific monoclonal antibodies against P-selectin, which blocked platelet-neutrophil adhesion. The results indicated that neutrophil inhibition of platelet reactivity was actually enhanced when adhesion was blocked (Figs. 5 and 6). Thus, neutrophils are more effective in controlling platelet reactivity when the cells are not adherent to each other. Thus, it should be considered that P-selectin, in keeping with other platelet $\alpha$-granule constituents, acts in a prothrombotic manner, via its ability to reduce neutrophil inhibition of platelet reactivity.

Two circumstances under which the efficiency of neutrophils in blocking platelet reactivity was increased included $(a)$ when lipoxygenase metabolism was blocked by ETYA in thrombin-stimulated cell suspensions and no hydroxy acids were produced (Fig. 3 ) and ( $b$ ) when metabolic platelet-neu- 
trophil adhesion was blocked by P-selectin antibodies (Figs. 5 and 6$)$. With regard to $(a)$, lipoxygenase-derived hydroxy acids have been reported to increase cell adhesion (40), and we could reduce neutrophil-mediated inhibition of platelet reactivity in vitro by adding back hydroxy acids (Table II). These compounds may have increased platelet adhesion. Thus, neutrophils may inhibit best when there is proximity, but not necessarily adhesion to stimulated platelets. We suggest that neutrophil inhibition of platelet reactivity may operate maximally via a fluid phase between the cells.

In conclusion, stimulation of platelets in the presence of neutrophils for $60 \mathrm{~s}$ results in inhibition of platelet reactivity. A P-selectin-induced adhesion step is not required for this downregulation mechanism. Furthermore, inhibition of P-selectininduced adhesion actually promoted neutrophil reduction of platelet responsiveness. Novel observations were made regarding participation of eicosanoid metabolites in these cell-cell interactions. The platelet 12-lipoxygenase product, 12S-HETE or its derivative, 12S,20-DiHETE, originating from transcellular metabolism between platelets and unstimulated neutrophils, reduced the inhibitory effect of neutrophils when the system was activated by thrombin. Also, aspirin treatment resulted in enhancement of the neutrophil downregulatory effect after thrombin stimulation. The latter represents a heretofore unrecognized potential antithrombotic effect of aspirin in the setting of thrombin formation in vivo. Neutrophil downregulation of platelet reactivity also occurred in platelet-rich plasma, indicating physiologic significance. These results demonstrate the role of neutrophils as cellular thromboregulators and provide further evidence for the concept of thrombosis as a multicellular process.

\section{Acknowledgments}

Drs. Bruce Furie, Barbara Furie, Susan Sajer, and Rodger McEver provided important critical discussions and generously shared invaluable antibodies for the research reported herein. We acknowledge the technical assistance of M. C. Insa, M. D. Lopez, and M. J. Solano. Ms. Evelyn M. Ludwig rendered expert editorial assistance.

This study was supported in part by grants from the Spanish "Fondo de Investigaciones Sanitarias de la Seguridad Social" 91 /0267 (J. Valles, M. T. Santos, J. Aznar), the Department of Veterans Affairs, and the National Institutes of Health (HL-29034 [M. J. Broekman] and HL-18828 (Specialized Center of Research in Thrombosis), HL46403 and HL-47073 [A. J. Marcus and M. J. Broekman]).

\section{References}

1. Bazzoni, G., E. Dejana, and A. Del Maschio. 1991. Platelet-neutrophil interactions. Possible relevance in the pathogenesis of thrombosis and inflammation. Haematologica. 76:491-499.

2. Selak, M. A., M. Chignard, and J. B. Smith. 1988. Cathepsin G is a strong platelet agonist released by neutrophils. Biochem. J. 251:293-299.

3. Ferrer-Lopez, P., P. Renesto, M. Schattner, S. Bassot, P. Laurent, and M. Chignard. 1990. Activation of human platelets by C5a-stimulated neutrophils: a role for cathepsin G. Am. J. Physiol. 258(Cell Physiol. 27):C1100-C1107.

4. Del Maschio, A., V. Evangelista, G. Rajtar, Z. M. Chen, C. Cerletti, and G. De Gaetano. 1990. Platelet activation by polymorphonuclear leukocytes exposed to chemotactic agents. Am. J. Physiol. 258 (Heart Circ. Physiol. 27):H870-H879.

5. Evangelista, V., G. Rajtar, G. De Gaetano, J. G. White, and C. Cerletti. 1991. Platelet activation by f MLP-stimulated polymorphonuclear leukocytes: the activity of cathepsin $G$ is not prevented by antiproteinases. Blood. 77:23792388.
6. Brower, M. S., R. I. Levin, and K. Garry. 1985. Human neutrophil elastase modulates platelet function by limited proteolysis of membrane glycoproteins. $J$. Clin. Invest. 75:657-666.

7. Zatta, A., M. Prosdocimi, V. Bertelé, G. Bazzoni, and A. Del Maschio. 1990. Inhibition of platelet function by polymorphonuclear leukocytes. J. Lab. Clin. Med. 116:651-660.

8. Schattner, M. A., J. R. Geffner, M. A. Isturiz, and M. A. Lazzari. 1990. Inhibition of human platelet activation by polymorphonuclear leukocytes. $\mathrm{Br}$. $\mathrm{J}$. Pharmacol. 101:253-256.

9. Nicolini, F. A., A. C. Wilson, P. Mehta, and J. L. Mehta. 1990. Comparative platelet inhibitory effects of human neutrophils and lymphocytes. $J$. Lab. Clin. Med. 116:147-152.

10. Nicolini, F. A., and J. L. Mehta. 1990. Inhibitory effect of unstimulated neutrophils on platelet aggregation by release of a factor similar to endotheliumderived relaxing factor (EDRF). Biochem. Pharmacol. 40:2265-2269.

11. Faint, R. W., I. J. Mackie, and S. J. Machin. 1991. Platelet aggregation is inhibited by a nitric oxide-like factor released from human neutrophils in vitro. Br. J. Haematol. 77:539-545.

12. May, G. R., P. Crook, P. K. Moore, and C. P. Page. 1991. The role of nitric oxide as an endogenous regulator of platelet and neutrophil activation within the pulmonary circulation of the rabbit. Br. J. Pharmacol. 102:759-763.

13. Santos, M. T., J. Valles, A. J. Marcus, L. B. Safier, M. J. Broekman, N. Islam, H. L. Ullman, A. M. Eiroa, and J. Aznar. 1991. Enhancement of platelet reactivity and modulation of eicosanoid production by intact erythrocytes. $J$. Clin. Invest. 87:571-580.

14. Valles, J., M. T. Santos, J. Aznar, A. J. Marcus, V. Martinez-Sales, M. Portoles, M. J. Broekman, and L. B. Safier. 1991. Erythrocytes metabolically enhance collagen-induced platelet responsiveness via increased thromboxane production, ADP release, and recruitment. Blood. 78:154-162.

15. Marcus, A. J. 1990. Eicosanoid interactions between platelets, endothelial cells, and neutrophils. Methods Enzymol. 187:585-598.

16. Broekman, M. J., A. M. Eiroa, and A. J. Marcus. 1991. Inhibition of human platelet reactivity by endothelium-derived relaxing factor from human umbilical vein endothelial cells in suspension. Blockade of aggregation and secretion by an aspirin-insensitive mechanism. Blood. 78:1033-1040.

17. Larsen, E., A. Celi, G. E. Gilbert, B. C. Furie, J. K. Erban, R. Bonfanti, D. D. Wagner, and B. Furie. 1989. PADGEM protein: a receptor that mediates the interaction of activated platelets with neutrophils and monocytes. Cell. 59:305-312.

18. Moore, K. L., A. Varki, and R. P. McEver. 1991. GMP-140 binds to a glycoprotein receptor on human neutrophils: evidence for a lectin-like domain. $J$. Cell Biol. 112:491-499.

19. Johnston, G. I., A. Kurosky, and R. P. McEver. 1989. Structural and biosynthetic studies of the granule membrane protein, GMP-140, from human platelets and endothelial cells. J. Biol. Chem. 264:1816-1823.

20. Geng, J.-G., M. P. Bevilacqua, K. L. Moore, T. M. McIntyre, S. M. Prescott, J. M. Kim, G. A. Bliss, G. A. Zimmerman, and R. P. McEver. 1990. Rapid neutrophil adhesion to activated endothelium mediated by GMP-140. Nature (Lond.). 343:757-760.

21. Hamburger, S. A., and R. P. McEver. 1990. GMP-140 mediates adhesion of stimulated platelets to neutrophils. Blood. 75:550-554.

22. Marcus, A. J., L. B. Safier, H. L. Ullman, M. J. Broekman, N. Islam, T. D. Oglesby, and R. R. Gorman. 1984. 12S,20-Dihydroxyicosatetraenoic acid: a new icosanoid synthesized by neutrophils from $12 \mathrm{~S}$-hydroxyicosatetraenoic acid produced by thrombin- or collagen-stimulated platelets. Proc. Natl. Acad. Sci. USA. 81:903-907.

23. Marcus, A. J., M. J. Broekman, L. B. Safier, H. L. Ullman, N. Islam, C. N. Serhan, L. E. Rutherford, H. M. Korchak, and G. Weissmann. 1982. Formation of leukotrienes and other hydroxy acids during platelet-neutrophil interactions in vitro. Biochem. Biophys. Res. Commun. 109:130-137.

24. Smith, G. P., and T. J. Peters. 1981. Subcellular localization and properties of adenosine diphosphatase activity in human polymorphonuclear leukocytes. Biochim. Biophys. Acta. 673:234-242.

25. Coade, S. B., and J. D. Pearson. 1989. Metabolism of adenine nucleotides in human blood. Circ. Res. 65:531-537.

26. Wright, C. D., A. Mülsch, R. Busse, and H. Osswald. 1989. Generation of nitric oxide by human neutrophils. Biochem. Biophys. Res. Commun. 160:813819.

27. McCall, T. B., N. K. Boughton-Smith, R. M. J. Palmer, B. J. R. Whittle, and S. Moncada. 1989. Synthesis of nitric oxide from L-arginine by neutrophils. Release and interaction with superoxide anion. Biochem. J. 261:293-296.

28. Schmidt, H. H. H. W., R. Seifert, and E. Bohme. 1989. Formation and release of nitric oxide from human neutrophils and HL-60 cells induced by a chemotactic peptide, platelet activating factor and leukotriene $\mathrm{B}_{\mathbf{4}}$. FEBS Lett. 244:357-360.

29. Berman, C. L., E. L. Yeo, J. D. Wencel-Drake, B. C. Furie, M. H. Ginsberg, and B. Furie. 1986. A platelet alpha granule membrane protein that is associated with the plasma membrane after activation. Characterization and sub- 
cellular localization of platelet activation-dependent granule-external membrane protein. J. Clin. Invest. 78:130-137.

30. McEver, R. P. 1991. Leukocyte interactions mediated by selectins. Thromb. Haemostas. 66:80-87.

31. Bevilacqua, M. P., and R. M. Nelson. 1993. Selectins. J. Clin. Invest. 91:379-387.

32. Johnston, G. I., R. G. Cook, and R. P. McEver. 1989. Cloning of GMP140 , a granule membrane protein of platelets and endothelium: sequence similarity to proteins involved in cell adhesion and inflammation. Cell. 56:1033-1044.

33. Bevilacqua, M. P., E. C. Butcher, B. C. Furie, B. Furie, M. Gallatin, M. A. Gimbrone, Jr., J. Harlan, K. Kishimoto, L. Lasky, R. P. McEver, et al. 1991. Selectins: a family of adhesion receptors. Cell. 67:233.

34. Watson, M. L., S. F. Kingsmore, G. I. Johnston, M. H. Siegelman, M. M. Le Beau, R. S. Lemons, N. S. Bora, T. A. Howard, I. L. Weissman, R. P. McEver, et al. 1990. Genomic organization of the selectin family of leukocyte adhesion molecules on human and mouse chromosome 1. J. Exp. Med. 172:263-272.

35. Moore, K. L., and L. F. Thompson. 1992. P-selectin (CD62) binds to subpopulations of human memory $\mathrm{T}$ lymphocytes and natural killer cells. Biochem. Biophys. Res. Commun. 186:173-181.
36. Packham, M. A., R. L. Kinlough-Rathbone, H.-J. Reimers, S. Scott, and J. F. Mustard. 1977. Mechanisms of platelet aggregation independent of adenosine diphosphate. In Prostaglandins in Hematology. M. J. Silver, J. B. Smith, and J. J. Kocsis, editors. Spectrum Publications, New York. 247-276.

37. Lawrence, M. B., and T. A. Springer. 1991. Leukocytes roll on a selectin at physiologic flow rates: distinction from and prerequisite for adhesion through integrins. Cell. 65:859-873.

38. Lorant, D. E., K. D. Patel, T. M. McIntyre, R. P. McEver, S. M. Prescott, and G. A. Zimmerman. 1991. Coexpression of GMP-140 and PAF by endothelium stimulated by histamine or thrombin: a juxtacrine system for adhesion and activation of neutrophils. J. Cell Biol. 115:223-234.

39. Palabrica, T., R. Lobb, B. C. Furie, M. Aronowitz, C. Benjamin, Y.-M. Hsu, M. A. Konstam, S. Sajer, and B. Furie. 1992. Leukocyte accumulation promoting fibrin deposition is mediated in vivo by P-selectin on adherent platelets. Nature (Lond.). 359:848-851.

40. Honn, K. V., I. M. Grossi, L. A. Fitzgerald, L. A. Umbarger, C. A. Diglio and J. D. Taylor. 1988. Lipoxygenase products regulate IRGpIIb/IIIa receptor mediated adhesion of tumor cells to endothelial cells, subendothelial matrix and fibronectin. Proc. Soc. Exp. Biol. Med. 189:130-135. 